\title{
Reabsorción radicular externa (RRE) asociada al tratamiento ortodóncico: revisión de la literatura
}

\author{
External root resorption as a result of orthodontic treatment: literature review
}

\author{
Daniela Alejandra Torres-Pinto ${ }^{1}$, María Catalina Álvarez-Parker ${ }^{2}$
}

Citación: Torres-Pinto DA, Álvarez-Parker MC. Reabsorción radicular externa (RRE) asociada al tratamiento ortodóncico: revisión de la literatura. Ustasalud 2020;19: 7-18.

Licencia Creative Commons

\section{Resumen}

La reabsorción radicular externa (RRE) se define como la destrucción del cemento o la dentina por la actividad cementoblástica u osteoclástica, dando como resultado un acortamiento de la raíz al ser sometida a una fuerza ortodóncica. Aproximadamente un $80 \%$ de los pacientes presentan algún grado de reabsorción. Esta condición tiene una gran variabilidad individual y su etiología es multifactorial, por lo que esta revisión de literatura tiene como propósito entregar información sobre el proceso de reabsorción radicular y sus factores de riesgo para poder prevenir o intervenir oportunamente este efecto indeseado. Para llevar a cabo este estudio se utilizaron bases de datos como Pubmed, Scielo, Wiley, Elsevier, teniendo como límite de búsqueda artículos publicados entre 2010 y 2020, a excepción de tres estudios, uno del 2005, del 2007 y otro del 2009, que proporcionaron información de suma relevancia sobre los factores de riesgo del fenómeno estudiado. La reabsorción radicular externa es una complicación prevalente y actualmente no existe un consenso para manejar este proceso, por eso son necesarios estudios sistemáticos que proporcionen evidencia más confiable para que, tanto ortodoncistas como otros especialistas, tengan más conocimiento del tema, evitando complicaciones en el diagnóstico, como durante el tratamiento al realizar movimientos dentales.

Palabras clave: Reabsorción radicular, tratamiento ortodoncia, factores predisponentes.

\begin{abstract}
External root resorption (RRE) is defined as a destruction of the cement or the dentin due to cementotic or osteoclastic activity, resulting in a shortening of the root when orthodontic force is applied. Approximately $80 \%$ of the patients present some degree of resorption. This condition has great variability, and its etiology is multifactorial, so this literature review aims to provide information of root resorption and its predisposing factors in order to prevent or intervene in a timely manner this undesired effect. To carry out this study, databases as Pubmed, Scielo, Wiley, Elsevier were used, having as a search limit articles published between 2010 and 2020, with the exception of three studies, one from 2005, from 2007 and the other one from 2009, which provided highly relevant information about risk factors of root resorption. External root resorption is a prevalent complication and there is currently no consensus to manage this phenomenon, which is why systematic studies are needed to provide more reliable evidence so that both orthodontists and other specialists have more knowledge of the subject and complications are avoid both in the diagnosis such as during treatment when performing tooth movements.
\end{abstract}

Keywords: Root resorption, orthodontic treatment, predisposing factors.
1 Cirujana dentista, licenciada en Odontología, Universidad de Valparaíso. Práctica Privada.

2 Cirujana dentista, licenciada en Odontología, Universidad Finis Terrae. Práctica Privada.

Autora de correspondencia: Daniela Alejandra Torres Pinto Correo electrónico: tanitorresp91@gmail.com
Recibido para publicación: 7 de julio de 2020

Aceptado para publicación

26 de noviembre de 2020 


\section{INTRODUCCIÓN}

La reabsorción radicular externa (RRE) es un proceso inflamatorio que resulta en necrosis del ligamento periodontal, se supera la capacidad reparativa del cemento permitiendo la degradación de la superficie radicular. Las células responsables de la reabsorción radicular son los odontoclastos, y se produce cuando los osteoclastos obtienen acceso al tejido mineralizado por alguna brecha en la capa de células formativas que cubre el tejido o cuando el precemento sufre algún tipo de daño mecánico; este proceso parece ser desencadenado por las citoquinas, por estas células inmunes que migran fuera de los capilares en el ligamento periodontal e interactúan con las células locales, elaborando una extensa cohorte de moléculas de señal, tales como la osteopontina y la sialoproteína ósea, para osteoblastos y osteoclastos.

El proceso de necrosis del ligamento periodontal durante el movimiento ortodóncico en el lado de presión con formación de zonas hialinas libres de células, seguida de reabsorción osteoclástica del hueso alveolar vecino y aposición de hueso por los osteoblastos en el lado donde existe tensión, son las características histológicas típicas de estos procesos. El proceso de la RRE continúa hasta que no haya tejido hialino o el nivel de fuerza producida por la ortodoncia decrezca. Las lagunas de reabsorción se expanden sobre las superficies radiculares involucradas y, por tanto, disminuyen indirectamente la presión ejercida a través de la aplicación de la fuerza. Esta pérdida de presión sobre los tejidos periodontales permite que se dé la reparación del cemento.

La RRE es considerada como un efecto colateral indeseable asociada a los movimientos ortodónticos ${ }^{1,2}$. Ottolengui (1993) fue quien estableció la relación directa entre el tratamiento ortodóncico y la RRE. La etiología exacta de esta patología es desconocida, pero es un problema en su mayoría multifactorial que compromete predisposición genética, factores ambientales asociados a la morfología radicular y factores mecánicos relacionados al tratamiento ortodóncico como el uso de fuerzas intensas ${ }^{3}$.

La reabsorción radicular asociada al tratamiento de ortodoncia es más evidente en los pacientes a quienes se les aplican fuerzas pesadas, de larga duración $y$ en direcciones desfavorables, o cuando el diente no es capaz de resistir las fuerzas fisiológicas, ya sea por factores como la presión de los dientes adyacentes, la inflamación periodontal o periapical, trauma oclusal o factores ideopáticos.

El grado promedio de reabsorción durante el tratamiento ortodóntico varía de 0,26 a 2,93 milímetros $(\mathrm{mm})$, en tanto que la prevalencia de la reabsorción detectable radiográficamente varía de $0 \%$ a $100 \%$, de acuerdo con Vlaskalic y Boyd ${ }^{1}$. Esas diferencias pueden atribuirse a diferencias considerables en el tipo de diente evaluado, tamaño de las muestras ${ }^{4}$, período de seguimiento ${ }^{5}$, tipo de movimiento dental y métodos de medición. Por lo tanto, la comparación de los estudios citados en la literatura es compleja ${ }^{1}$.

El propósito de la presente revisión de la literatura es brindar información específica, tanto del proceso de RRE inducida por movimientos ortodóncicos, como de los factores que predisponen la aparición de esta patología, para que así el clínico pueda desarrollar estrategias con el fin de prevenir o interceptar oportunamente la RRE.

\section{MÉTODOS}

Se hizo una revisión, a través de la búsqueda en las siguientes bases de datos: Pubmed, Scielo, Wiley, Elsevier, Oxford Journals y Science. De estas fuentes se utilizaron artículos de texto completo y se seleccionaron con base en el nivel de evidencia y calidad de los reportes. Así mismo, se incluyeron las revistas científicas suscritas y de libre acceso encontradas en las bases de datos. Se buscaron revisiones sistemáticas, estudios clínicos, estudios de casos y controles, de cohorte y transversales.

Dentro de los criterios de inclusión, los artículos debían contener las palabras de búsqueda, tener acceso al texto completo, ser publicaciones en español o inglés, y limitarse a los últimos 10 años, a excepción de tres estudios que son del 2005, 2007 y 2009. Por otra parte, fueron excluidos aquellos artículos que estuviesen en un idioma diferente a los anteriormente descritos, o que no existiera la posibilidad de obtener el texto completo.

Las palabras clave utilizadas fueron reabsorción radicular, reabsorción radicular externa, tratamiento ortodoncia, factores predisponentes. Para definir el 
nivel de evidencia científica y grado de recomendación se utilizó el esquema propuesto por la Agència d'Avaluació de Tecnologia Mèdica (AATM).

\section{RESULTADOS}

Luego de aplicar los criterios de inclusión y exclusión, 32 artículos fueron seleccionados, entre ellos revisiones sistemáticas, estudios clínicos, estudios de casos y controles, de cohorte y transversales.

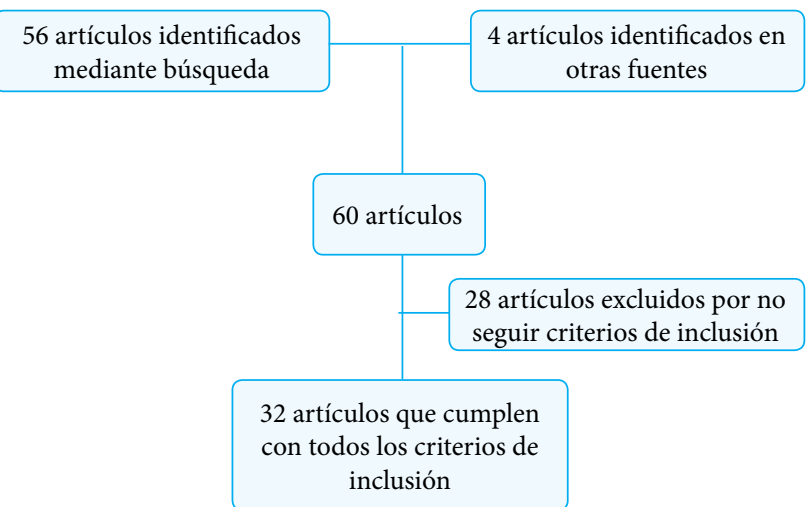

Figura 1. Diagrama de flujo que indica el número de artículos incluidos y excluidos.

\section{Reabsorción radicular: definición}

La reabsorción radicular (RR) de un diente permanente es un proceso patológico de destrucción progresivo de carácter idiopático que ocurre en la dentina de la cámara pulpar o de los conductos radiculares. Este tipo de reabsorción es conocido como reabsorción radicular interna (RRI).

Si el proceso se presenta en la superficie externa de la raíz, se denomina reabsorción radicular externa (RRE), estos fenómenos pueden provocar la pérdida del diente y es probablemente la secuela más común del tratamiento ortodóncico. La RRE es un proceso impredecible, y cuando alcanza la dentina se considera que el proceso es irreversible, causando incluso la pérdida de uno o más dientes.

La reabsorción radicular externa ocurre cuando la capa cementoblástica o cualquier tejido de la superficie dental es dañada o removida6, ya sea por un estímulo mecánico (tratamiento de ortodoncia o trauma dentoalveolar) o químico (blanqueamiento dental)1. Es un proceso inflamatorio que resulta en necrosis del ligamento periodontal cuando se aplica una fuerza ortodóncica intensa o descontrolada. La reabsorción ocurre cuando la presión aplicada en el cemento radicular excede su capacidad reparativa y la dentina es expuesta, permitiendo que los odontoclastos degraden la superficie radicular 7 .

\section{Tipos de reabsorción radicular}

Andreasen en 1985 clasificó la reabsorción radicular según causa traumática en ${ }^{6}$ :

- Reabsorción superficial: Se trata de un estado transitorio en el que se produce una destrucción mínima que se repara espontáneamente por un tejido de cemento-hueso en donde quedan incluidas las células clásticas en dicho tejido mineralizado.

- Reabsorción inflamatoria: Resulta de la combinación entre la agresión al ligamento periodontal y la superficie radicular como consecuencia de una agresión, hay presencia de bacterias dentro del conducto radicular, las cuales, a su vez, estimulan la actividad clástica.

- Reabsorción por sustitución (anquilosis): Consecuencia de la anquilosis entre hueso y diente por lo que no hay ligamento periodontal interpuesto, y el hueso en remodelación constante elimina poco a poco el diente y lo sustituye gradualmente ${ }^{8}$.

La reabsorción radicular debida al tratamiento ortodóncico, según la clasificación de Andreasen, es del tipo reabsorción superficial o reabsorción inflamatoria. En el primer caso, se trata de un proceso autolimitado de la superficie externa de la raíz; y en el segundo, el proceso de reabsorción es dependiente de una estimulación continua o reestimulación de las células odontoclásticas por infección o presión.

\section{Factores etiopatogénicos}

La naturaleza de las reabsorciones radiculares no está completamente esclarecida, pero parece haber una dependencia con factores relacionados con el paciente y con el tratamiento ortodóncico?. 
Dentro de los factores relacionados con el paciente encontramos factores sistémicos y factores relacionados con las características propias del diente. Dentro de los factores sistémicos se encuentran: genéticos, sistémicos, como hipotiroidismo, alergias y asma, el consumo de medicamentos, tales como l-tiroxina, bifosfonatos y bajas dosis de corticoides; la edad, género, los malos hábitos como la onicofagia, la interposición lingual y consumo de alcohol ${ }^{1}$. Dentro de los factores asociados al diente se encuentran: la estructura facial y dentoalveolar, dentro de las cuales están la densidad del hueso alveolar y la proximidad de las raíces con la cortical ósea, el tipo de diente, la morfología radicular, la vitalidad pulpar, una reabsorción radicular o un trauma dentoalveolar previo, una infección periapical, algún tipo de maloclusión como exceso vertical y mordidas abiertas, aplasia ${ }^{10} y$ tipo de diente $e^{9,11,12}$.

Dentro de los factores relacionados con el tratamiento ortodóncico se encuentran: magnitud de la fuerza ortodóncica, el tipo de fuerza, la dirección del movimiento dental, el tipo de aparatología ortodóncica, la duración del tratamiento ${ }^{11}$ y las extracciones dentales por indicación ortodóncica ${ }^{2,9}$.

\section{Epidemiología}

La reabsorción radicular externa es un fenómeno común asociado al tratamiento de ortodoncia y puede comenzar en las primeras etapas del tratamiento. Aproximadamente en un $80 \%$ de los pacientes sometidos a tratamiento de ortodoncia sufren de algún grado de $\mathrm{RRE}^{13}$. Los valores medios oscilan entre 0.5 y $3 \mathrm{~mm}$. de acortamiento radicular durante el tratamiento ${ }^{11}$, medidas obtenidas a partir de radiografías periapicales. Estudios histológicos indican una ocurrencia extremadamente alta (más del 90\%) de reabsorción radicular causada por fuerzas ortodóncicas ${ }^{4,5}$. Particularmente, los dientes afectados con mayor frecuencia y severidad son los incisivos laterales ${ }^{14,15}$, debido a que tienen un $30 \%$ de probabilidad de presentar una curvatura en su raíz y de este porcentaje un $66.6 \%$ que la curvatura se presente hacia distal.

Entre un $48 \%$ y $66 \%$ de los dientes tratados ortodóncicamente muestran una leve a moderada reabsorción radicular ${ }^{16}$. Respecto a la RR severa, algunos estudios que estiman el desarrollo de esta entre un $1-5 \%$ de los dientes ${ }^{4,5}$. Por el contrario en estudios se observaron que afecta hasta un $15 \%$ de los pacientes tratados ortodóncicamente ${ }^{17}$.

Es casi imposible estimar la incidencia, prevalencia o el grado de severidad de reabsorción radicular debido a la diferencia de metodología entre los estudios publicados ${ }^{13}$.

\section{Diagnóstico}

El diagnóstico precoz es un factor crítico en el manejo de la reabsorción radicular, ya que, si se trata de manera oportuna, menores serán las consecuencias. El diagnóstico debe basarse en un examen clínico y radiográfico ${ }^{6}$. Actualmente no existe un método de elección ideal para detectar la reabsorción radicular ${ }^{9}$.

Tabla 1. Factores de riesgo para reabsorción radicular

\begin{tabular}{ll}
\hline \multicolumn{2}{c}{ Factores de riesgo relacionados con el paciente } \\
\hline Factores genéticos & Morfología radicular \\
Factores sistémicos & Vitalidad - sensibilidad pulpar \\
Consumo de medicamentos & Reabsorción radicular previa \\
Edad cronológica & Traumatismo dentoalveolar previo \\
Género & Tipo o severidad de la maloclusión \\
Consumo de alcohol & Estructura facial y dentoalveolar \\
Malos hábitos & Aplasia \\
& Tipo de diente \\
\hline \multicolumn{1}{c}{ Factores de riesgo relacionados con el tratamiento ortodóncico } \\
\hline Magnitud de la fuerza ortodóncica & Duración del tratamiento \\
Tipo de fuerza & Extracciones dentales por indicación \\
Dirección del movimiento dental & ortodóncica. \\
Tipo de aparatología ortodóncico & \\
\hline
\end{tabular}


Sin embargo, la reabsorción radicular posterior a un tratamiento de ortodoncia ha sido analizada por muchos años con radiografías convencionales (periapicales, panorámicas y cefalometría $)^{2,9,11}$. Estudios recientes indican que la tomografía computarizada o el Cone Beam (CBCT) podrían ser herramientas útiles para detectar la RRE'

Las radiografías periapicales obtenidas con la técnica del paralelismo son más exactas, debido a que generan un menor grado de distorsión geométrica de los dientes ${ }^{2}$, no ocurre lo mismo con las radiografías panorámicas que tienen como limitación la superposición de las estructuras óseas en los dientes anteriores ${ }^{5,15}$.

Levander y Malmgren (1998) clasificaron la reabsorción radicular a partir de una radiografía periapical en:

- Nivel 1: La reabsorción es mínima, muestra un leve contorno irregular en el ápice y se mantiene la longitud radicular.

- Nivel 2: La reabsorción no es mayor a $2 \mathrm{~mm}$ en los tejidos duros.

- Nivel 3: La reabsorción destruye hasta $1 / 3$ de la raíz.

- Nivel 4: La reabsorción se extiende más allá del $1 / 3$ de la raíz ${ }^{18,19,20}$.
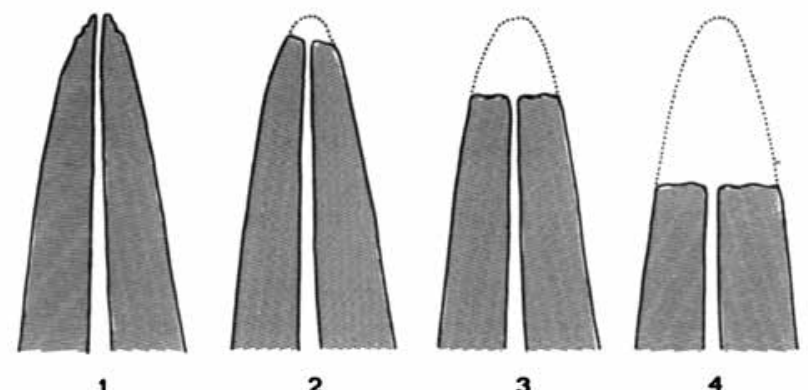

Figura 2. Índice de reabsorción radicular para su evaluación cuantitativa.

Fuente: Levander E, Malmgren O. Evaluation of the risk of root resorption during orthodontic treatment: a study of upper incisors. Eur J Orthod. 1988;10:30-8.

Respecto a la cefalometría, esta es el mejor método para comparar la longitud de las raíces de incisivos antes y después del tratamiento. Sin embargo, en pa- cientes con apiñamiento anterior se dificulta ubicar la posición de los ápices antes del tratamiento, además existe la superposición de las hemiarcadas ${ }^{2,5,9}$.

Estudios indican que la tomografía computarizada Cone Beam es el método diagnóstico más eficaz para detectar la reabsorción radicular, ya que presenta una alta sensibilidad y especificidad, proporcionando una visión en tres dimensiones ${ }^{4,5,11}$. Sin embargo, el principal problema es la alta dosis de radiación que recibe el paciente y el costo económico ${ }^{9,21}$.

\section{Manejo y recomendaciones}

La prevención va encaminada a vigilar los factores de riesgo que pueden estar bajo el control del ortodoncista, es decir, los mecánicos relacionados al tratamiento. La terapia ortodóncica en aquellos pacientes considerados de alto riesgo de RRE, ya sea por susceptibilidad individual, morfología radicular o factores sistémicos, debe ser cuidadosamente planificada?

Actualmente no existe un consenso sobre el manejo de los diferentes tipos de $\mathrm{RRE}^{6}$, pero se pueden dar ciertas recomendaciones basadas en la evidencia.

1. Previas al tratamiento de ortodoncia:

- Es importante realizar una buena anamnesis para identificar cualquier factor de riesgo para $\mathrm{RRE}^{4,9,18}$.

- Frecuentemente existe cierto grado de reabsorción que es inherente al tratamiento de ortodoncia, es por esto por lo que los pacientes deben estar informados sobre esta posible complicación ${ }^{4,13,18}$. En el caso de que se planeen extracciones dentales, informar al paciente sobre el aumento en el riesgo de sufrir RRE severa ${ }^{16}$.

- Debido a que generalmente las RRE son asintomáticas, las imágenes radiográficas constituyen la mejor manera de detectar y medir la severidad para obtener un diagnóstico temprano. La radiografía panorámica es la más usada en el diagnóstico ortodóncico debido a que muestra todo el complejo maxilo-mandibular, incluyendo las articulaciones temporo-mandibulares, pero en 
el caso de que se sospeche de RRE en el sector anterior se recomienda tomar una radiografía periapical porque presenta menor distorsión en la imagen ${ }^{16}$. Se indican controles radiográficos después de 6-12 meses de iniciado el tratamiento ortodóncico ${ }^{9,18,19,22}$ y en aquellos dientes de riesgo aumentado cada 3 meses ${ }^{9}$.

- El tratamiento, siempre que sea posible, ha de iniciarse cuanto más joven es el paciente mejor ${ }^{3,9}$, esto se explica porque a mayor edad se reduce la vascularidad de la membrana periodontal y la densidad ósea $1^{1}$. Además, los dientes de pacientes jóvenes presentan tejido cementoide sobre la superficie radicular y las células clásticas no atacan la predentina no calcificada ${ }^{1}$.

2. Durante el tratamiento de ortodoncia:

- Se recomienda planificar un tratamiento ortodóncico con una duración adecuada (menos de tres años) $)^{13 .}$

- Se recomienda emplear fuerzas ligeras (menores a $150 \mathrm{gr} / \mathrm{cm}^{2}$ ) y controladas, es decir, que permitan el tiempo suficiente para que haya reabsorción de la zona hialina y una adecuada recuperación de los tejidos, además se recomienda comenzar por arcos de bajo calibre que proporcionen fuerzas ligeras ${ }^{3,4,9,13}$, como por ejemplo arcos de níquel titanio termo-activados.

- Son recomendables intervalos superiores a cuatro semanas entre activaciones ${ }^{4,9}$.

- Ante una reabsorción localizada o en casos de compromiso pulpar, se recomienda realizar una interconsulta al endodoncista para evaluar la necesidad de realizar un tratamiento de conductos, una cirugía periapical o la medicación intra-conducto, durante lo que resta de tratamiento. En la mayoría de los casos no es necesario interrumpir el tratamiento ortodóncico ${ }^{6,9}$.

- En el caso de que se diagnostique una RRE se recomienda pausar el tratamiento por un periodo de dos a tres meses con un arco de alambre pasivo $^{13,15,19,22}$, ya que la reparación total de la reabsorción radicular ocurre a las 8 semanas $^{23}$. Si se decide continuar con el tratamiento de manera activa luego de la pausa de 3 meses es mandatorio solicitar una nueva radiografía a los 6 meses $^{16}$.

- Si durante el tratamiento se presenta una RRE de moderada a severa, esto implica un replanteamiento del plan de tratamiento para evitar movilidad excesiva e incluso pérdida dental ${ }^{9,13,18,22}$.

- Si la RRE es severa, se deben buscar nuevas alternativas terapéuticas a la ortodoncia, dentro de las cuales se podrían incluir, el cierre de espacios mediante soluciones protéticas, el stripping en vez de realizar extracciones y ferulización de los dientes con $\mathrm{RRE}^{4,9,22}$ con férula flexible, ya que presentan mayor estabilidad en el tiempo ${ }^{9}$ y evitan que se produzca anquilosis en la raíz.

3. Después del tratamiento de ortodoncia:

- Siempre se debe solicitar una radiografía final post-tratamiento ${ }^{9,16} \mathrm{y}$ realizar seguimientos de acuerdo con el criterio del profesional ${ }^{16}$.

- Si se diagnostica RRE en las radiografías finales después del tratamiento, se debe realizar un seguimiento hasta que la RRE se detenga ${ }^{9,18}$. Si se mantiene se debe considerar una terapia con hidróxido de calcio ${ }^{22}$ o la endodoncia de los dientes comprometidos ${ }^{9}$ para eliminar el tejido de granulación y rellenar el defecto de reabsorción.

- El odontólogo debe mantener una buena comunicación con sus pacientes posterior al tratamiento, realizar chequeos para evitar una posible enfermedad periodontal y revisar que las contenciones permanezcan en buen estado ${ }^{4,16,18}$.

\section{Discusión}

La reabsorción radicular es considerada una de las principales secuelas del tratamiento ortodóncico ${ }^{24}$. Sin embargo, en todos los tratamientos se presenta 
una ligera reabsorción radicular o remodelado de la raíz, el cual es difícil evidenciar radiográficamente y se dificulta aún más si no es realizado con buenos criterios de evaluación; esto puede llevar a muchos errores, que se minimizan utilizando radiografías estandarizadas tomadas con el método del paralelismo ${ }^{24}$. Maués et al. indican que las radiografías periapicales son las más utilizadas para detectar esta complicación, debido a su exactitud comparada con la radiografía panorámica y es mejor la relación costo-beneficio en comparación con el Cone Beam o $(\mathrm{CBCT})^{12}$. Sin embargo, en varios estudios se concluye que el Cone Beam es el método diagnóstico más eficaz para detectar la RR ya que presenta una alta sensibilidad y especificidad, además proporciona una visión en los tres planos del espacio ${ }^{4,5,11}$.

La etiología de la reabsorción radicular no está claramente definida y es muy compleja, ya que involucra muchos factores de riesgo, en cuanto a los asociados al paciente, Abuabara (2007) indica que hay un factor genético para RRE, ya que existe una asociación entre IL-1 y TNF y la RR al ser citoquinas proinflamatorias ${ }^{14}$. Luna y colaboradores (2011) exponen que una baja producción de IL-1B aumenta el riesgo de producir este fenómeno durante el tratamiento de ortodoncia ${ }^{3}$.

Existe una mayor prevalencia de reabsorción radicular y pacientes alérgicos o asmáticos ${ }^{1,14,25}$ ya que se producen ciertos mediadores inflamatorios como la interleuquina ${ }^{4,5,6,10 \text { y } 13}$ responsables de $\mathrm{RRE}^{8,25}$. La administración de suplementos tiroideos beneficia a los pacientes en la fase inicial de la reabsorción radicular, al detener en muchas ocasiones el proceso. La hormona tiroidea influencia la actividad osteoclástica y estimula la reabsorción alveolar por medio de sustancias como la tiroxina ${ }^{1}$. El consumo de alcohol tiende a aumentar la RRE como consecuencia de la hidroxilación en el hígado de la vitamina $\mathrm{D}^{1,9}$. Las dosis altas de corticoides $(15 \mathrm{mg} / \mathrm{kg})$, promueven esta condición, mientras que la administración de dosis bajas $(1 \mathrm{mg} / \mathrm{kg}$ ) actúan como un factor protector; esto se explica, ya que los corticoides en dosis elevadas interfieren en el acoplamiento del ciclo aposición - reabsorción del hueso, lo que se traduce como una reducción en la producción de hueso y un aumento de la reabsorción ósea, además, se ha visto que retarda la remodelación de las fibras de colágeno, en cambio, a dosis bajas, existe una menor cantidad de la enzima fosfatasa ácida resistente al tartrato (TRAP), considerada un marcador histoquímico de los osteoclastos, generando una ac- ción supresora sobre la actividad clástica. La prednisona tiene efecto inhibitorio, al igual que los bifosfonatos, el celecoxib y algunos antibióticos como la doxicilina ${ }^{1,22}$.

Sondeijker y colaboradores exponen que la evidencia indica como baja o muy baja para todos los factores de riesgo relacionados con el paciente a excepción de la edad y el género que indica que no tiene relación con $\mathrm{RRE}^{16}$. Pastro y colaboradores concuerdan con que no existe relación entre el género y edad y la reabsorción radicular, pero sí habría relación en el caso de que un paciente adulto presentara periodontitis crónica ${ }^{20}$. Sin embargo, Vaquero y colaboradores indican en su estudio que las mujeres son más propensas a sufrir de RRE9 .

Respecto a la estructura facial y dentoalveolar, a mayor densidad del hueso alveolar se produce más frecuentemente RR. El contacto de las raíces con la cortical ósea durante el desplazamiento dentario aumentan el riesgo de lesión radicular ${ }^{1,9}$.

Existen controversias respecto a los malos hábitos y la aparición de reabsorción radicular. Luna y colaboradores indican que malos hábitos como la onicofagia, el empuje lingual con mordida abierta, la succión digital y la presión lingual son factores de riesgo para la RRE, Abuabara, también reporta como factor de riesgo la onicofagia, la interposición lingual y la interposición labial ${ }^{14}$, sin embargo, Pastro y colaboradores encontraron que solo la onicofagia es un factor de riesgo ${ }^{20}$.

La mayoría de los estudios coinciden que los incisivos laterales superiores son los dientes más propensos a sufrir de RRE debido a la extensión del movimiento durante el tratamiento y presentan mayor probabilidad de tener raíces con dilaceración hacia distal ${ }^{26}$. Respecto a la morfología radicular, raíces cortas, dilaceradas, puntiagudas o con forma de pipeta son más propensas a reabsorberse, porque distribuyen de manera diferente las fuerzas ${ }^{1,3,15,25}$, al aplicar una fuerza a nivel del ápice, la distribución de la carga es diferente a la que se genera en una raíz con una anatomía radicular normal, causando un trauma al ligamento periodontal ${ }^{3}$.

Una de las principales preguntas que surgen, es si los dientes tratados endodónticamente tienen una mayor probabilidad de reabsorción con relación a los dientes sin tratamiento de conductos previo. Lozano y colaboradores afirman que los dientes tratados en- 
dodónticamente previo a la ortodoncia se reabsorben menos que los dientes vitales debido a que estos últimos tienen un paquete vasculonervioso, que le otorga junto al ligamento periodontal una mayor capacidad, adicionalmente estos dientes con endodoncia presentan una mayor dureza y densidad en la dentina ${ }^{1}$, esto confirmaría la teoría que un diente con TDA previo, con afectación de la vitalidad pulpar, presenta menor riesgo de RRE. Sin embargo, Dindaroğlu y colaboradores mencionan que existe poca evidencia que compruebe esto en seres vivos ${ }^{11}$. Se recomiendan nuevas investigaciones sobre el manejo ortodóncico y endodóntico en casos de trauma dentoalveolar y RRE.

Otro factor de riesgo de RRE es la reabsorción radicular previa, Pastro y colaboradores encontraron que los dientes que presentan RRE previo a la ortodoncia son más propensos a sufrir RRE severa ${ }^{20}$, Lozano y colaboradores indican que las reabsorciones previas se incrementan entre $4 \%$ y $70 \%$ después del tratamiento ortodóncico ${ }^{1}$.

De acuerdo con la maloclusión, Fang y colaboradores en su estudio comprobaron que sí es un factor de riesgo para la reabsorción radicular ${ }^{25}$, ya que está relacionada por la mayor cantidad de movimiento dental necesario en el tratamiento ortodóncico ${ }^{3,20}$. En cuanto al tipo de maloclusión, algunos autores indican que un exceso vertical y las mordidas abiertas tienden a presentar mayores índices de $\mathrm{RRE}^{1}$, otros indican que las mordidas abiertas y un overjet aumentado son más propensos ${ }^{9}$, Maués y colaboradores obtuvieron como resultado que un overjet superior a $5 \mathrm{~mm}$ es un factor de riesgo para RRE severa.

Respecto a los factores de riesgo asociados al tratamiento ortodóncico, una magnitud de fuerza mayor a $150 \mathrm{gr} / \mathrm{cm}^{2}$ induce significativamente un mayor riesgo de sufrir RRE que las fuerzas ligeras ${ }^{3,4,7,711,18,22,24,27,28,29}$. Las fuerzas continuas y controladas que se activan cada 4 semanas que permiten la recuperación biológica, generan menos reabsorción en comparación con las fuerzas intermitentes o descontroladas propiciadas, por ejemplo, por aparatos removibles ${ }^{3,4,7,9,11,14,17,18,22,23,28}$. Cabe destacar que si se hace en el tiempo correcto siempre hay una recuperación biológica total, por lo que si no hay factores etiológicos asociados no habrá RRE. En cuanto a la dirección del movimiento dental, los movimientos de intrusión y torque generan mayor reabsorción radicu$\operatorname{lar}^{1,4,7,9,22}$, se ha comprobado en múltiples estudios que los movimientos de intrusión generan cuatro veces más reabsorción que los movimientos de extrusión ${ }^{13,14,17,23}$, esto se explica, ya que en los movimientos intrusivos, no toda la fuerza se distribuye a lo largo de la superficie radicular, sino que se concentra a nivel del ápice radicular ${ }^{12}$. Cabe mencionar que los movimientos intrusivos pueden producir la compresión de los vasos sanguíneos que penetran por el foramen apical, llegando a ocasionar la desvitalización de los diferentes elementos celulares del tejido pulpar ${ }^{30}$.

Respecto al tipo de aparato ortodóncico, Lozano et al. informan que la aparatología removible afecta más a las raíces que la aparatología fija, ya que existe un menor control de las fuerzas1, situación que no es corroborada por Vlasa y colaboradores, quienes indican que la aparatología fija genera mayor reabsorción porque existe mayor movimiento dental ${ }^{7}$. El uso de alambres rectangulares (provocan mayor fuerza) y de elásticos intermaxilares están relacionados significativamente con la severidad de la $\mathrm{RRE}^{1,2,10}$, Motokawa y colaboradores en su estudio obtuvieron como resultado una mayor prevalencia de RRE en el grupo que usó elásticos clase II por más de 6 meses $^{31}$. Los alineadores removibles (invisalign) no generan mayor predisposición a este fenómeno en comparación con la aparatología fija, esto puede deberse a que los protocolos actuales del uso de aparatología fija usan fuerzas ligeras en cada paso ${ }^{11,16,29,32}$, Fang y colaboradores exponen que si existe la colaboración del paciente y un tratamiento bien diseñado por parte del ortodoncista (alineadores removibles), se pueden predecir los movimientos, evitando movimientos repetidos y reduciendo la posibilidad de que se produzca esta complicación al ser comparada con la aparatología fija ${ }^{27}$.

En cuanto a la duración del tratamiento ortodóncico, una mayor duración podría ser un factor de riesgo para RRE debido a la extensión de los movimientos ${ }^{1,2,7,9,10,11,21,29}$ y porque además tiene un efecto negativo en la higiene oral ${ }^{16}$, sin embargo, Fang y colaboradores encontraron que la duración del tratamiento no es un factor coadyuvante ${ }^{27}$.

Las exodoncias dentales por razones ortodóncicas son un factor de riesgo para reabsorción radicular externa ${ }^{3,22,26}$ porque implica mayor extensión de movimiento dental, y de mayor fuerza para lograr la retrusión de las raíces para cerrar los espacios ${ }^{9,16,20,31}$. 


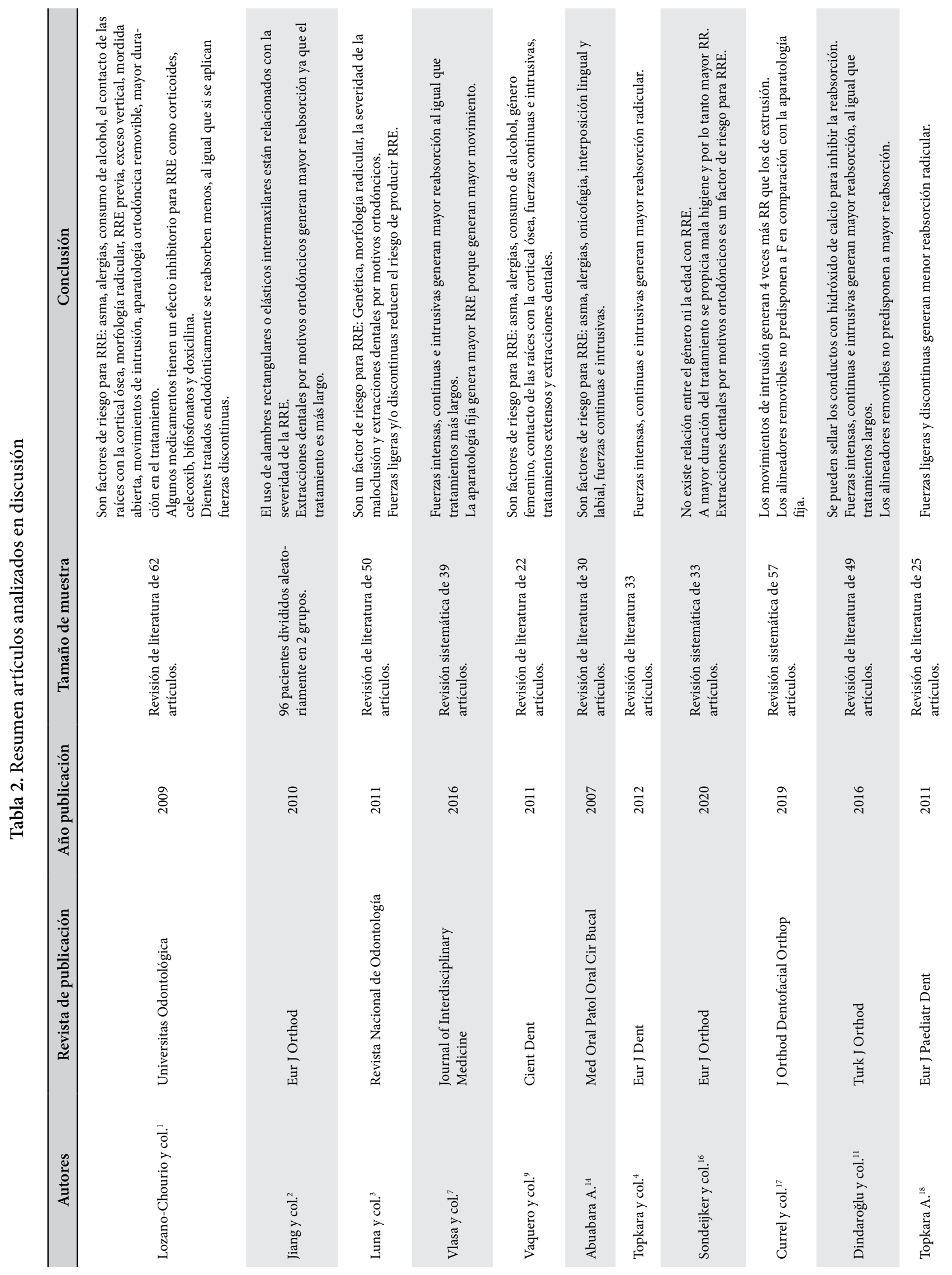




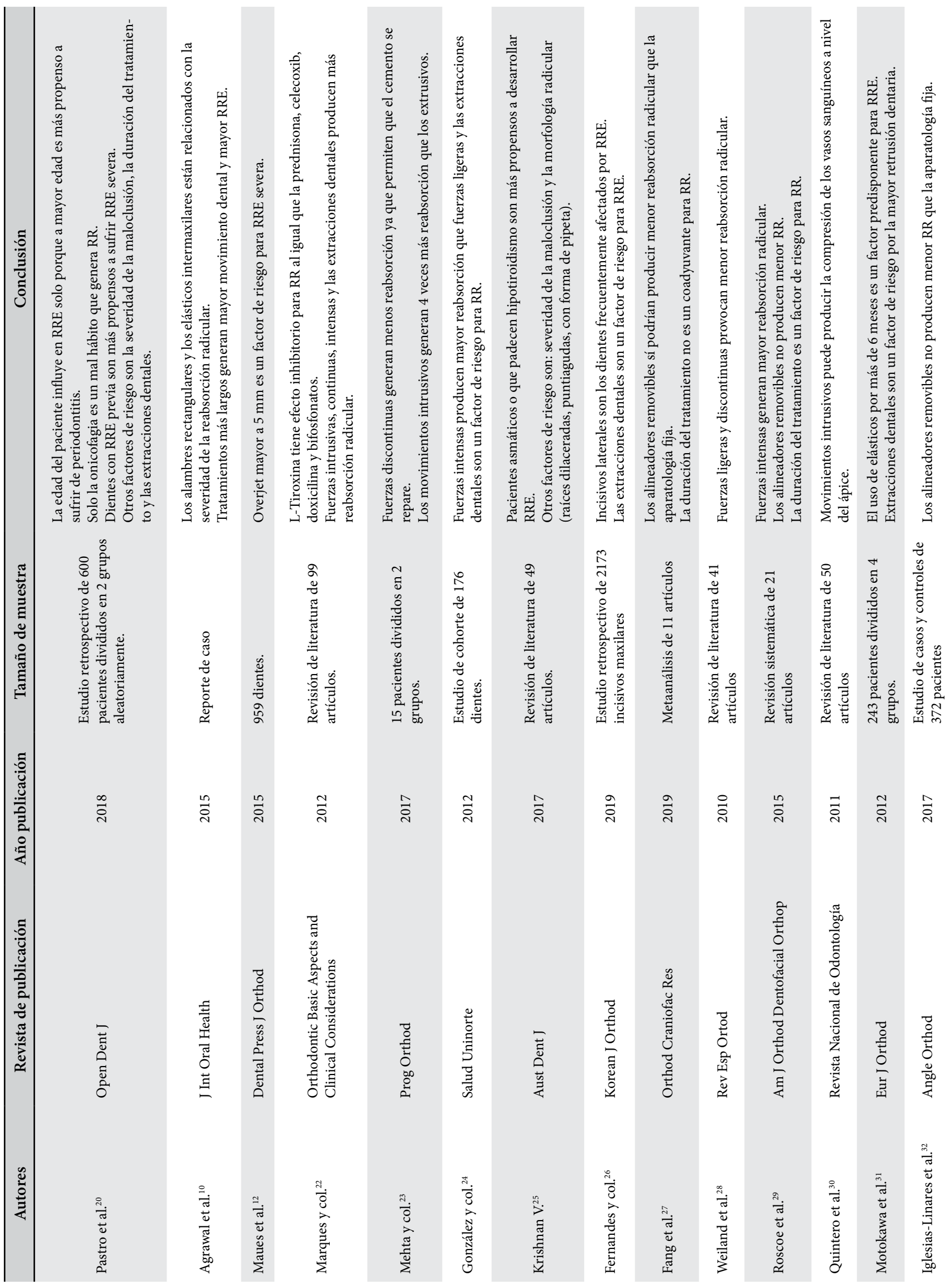




\section{CONCLUSIONES}

Es de suma importancia que el ortodoncista tenga el conocimiento suficiente de cómo y por qué se produce la reabsorción radicular, los factores de riesgo tanto biológicos como mecánicos, para prevenir la aparición de esta complicación. Según la evidencia científica, el método más utilizado para diagnosticar RR sigue siendo la radiografía, pero nuevas alternativas han surgido, como el Cone Beam o (CBCT) que permite obtener una imagen en 3 planos del espacio. Se requieren nuevos estudios sistemáticos que proporcionen evidencia más confiable sobre la prevalencia, los factores de riesgo y el manejo de la reabsorción radicular para saber cómo prevenir o intervenir oportunamente.

\section{REFERENCIAS}

1. Lozano-Chourio MA, Ruiz Rojas AL. Reabsorción radicular en ortodoncia: Revisión de la literatura. Univ Odontol 2009; 28(60): 45-52.

2. Jiang RP, McDonald JP, Fu MK. Root resorption before and after orthodontic treatment: a clinical study of contributory factors. Eur J Orthod. 2010;32(6):693-697. DOI: 10.1093/ejo/cjp165.

3. Luna C, Sánchez R, Zapata E, Rendón J. Reabsorción radicular asociada a movimientos ortodóncicos: una revisión de literatura. Revista Nacional de Odontología 2011;7(13):61-7.

4. Topkara A, Karaman AI, Kau CH. Apical root resorption caused by orthodontic forces: A brief review and a longterm observation. Eur J Dent. 2012;6(4):445-453.

5. Márquez JF, Castaño JM, Rueda ZV, Rendón J. Diagnóstico de reabsorción radicular externa en ortodoncia. Una revisión sistemática. Revista Nacional de Odontología. 2012;8(14):62-75.

6. Ahangari Z, Nasser M, Mahdian M, Fedorowicz Z, Marchesan MA. Interventions for the management of external root resorption. Cochrane Database of Systematic Reviews 2015, Issue 11. Art. No.: CD008003. DOI: 10.1002/14651858.CD008003.pub3.

7. Vlasa A, Yero L, Lazar L, Bud L, Pacurar M, Bud E, Biris C. Correlation between orthodontic forces and root resorption - a systematic review of the literature. Journal of Interdisciplinary Medicine 2016;1(2):142-145. DOI: 10.1515/jim-2016-0033.

8. Serrano Pardo S, Mendoza Rodríguez M, Márquez Rodríguez S, Pontigo Loyola A, Medina Solís C. Resorción radicular en ortodoncia. Revisión bibliográfica. Educación y Salud Boletín Científico Instituto de Ciencias de la Salud Universidad Autónoma del Estado de Hidalgo, 2017;5(9). DOI: $10.29057 /$ icsa.v5i9.2254.

9. Vaquero P, Perea B, Labajo E, Santiago A, García F. Reabsorción radicular durante el tratamiento ortodóncico: causas y recomendaciones de actuación. Cient Dent 2011;8(1):61-70.

10. Agrawal CM, Mahida K, Agrawal CC, Bothra J, Mashru $\mathrm{K}$. A rare case of apical root resorption during orthodontic treatment of patient with Multiple Aplasia. J Int Oral Health. 2015;7(7):124-127.

11. Dindaroğlu F, Doğan S. Root resorption in orthodontics. Turk J Orthod. 2016;29(4):103-8. DOI: 10.5152/ TurkJOrthod.2016.16021.

12. Maués CPR, Nascimento RR, Vilella OV. Severe root resorption resulting from orthodontic treatment: Prevalence and risk factors. Dental Press J Orthod. 2015;20(1):52-8. DOI: 10.1590/2176-9451.20.1.052-058.oar.

13. Feller L, Khammissa R, Thomadakis G, Fourie J, Lemmer J. Apical external root resorption and repair in orthodontic tooth movement: Biological events. BioMed Research International 2016(2):1-7. DOI: 10.1155/2016/4864195.

14. Abuabara A. Biomechanical aspects of external root resorption in orthodontic therapy. Med Oral Patol Oral Cir Bucal. 2007;12(8):E610-3.

15. Smale I, Artun J, Behbehani F, Doppel D, van't Hof M, Kuijpers-Jagtman AM. Apical root resorption 6 months after initiation of fixed orthodontic appliance therapy. Am J Orthod Dentofacial Orthop. 2005;128(1):57-67. DOI: 10.1016/j.ajodo.2003.12.030.

16. Sondeijker CFW, Lamberts AA, Beckmann SH, Kuitert $\mathrm{RB}$, van Westing $\mathrm{K}$, et al. Development of a clinical practice guideline for orthodontically induced external apical root resorption. Eur J Orthod. 2020;42(2):115-124. DOI: 10.1093/ejo/cjz034.

17. Currell SD, Liaw A, Blackmore Grant PD, Esterman A, Nimmo A. Orthodontic mechanotherapies and their influence on external root resorption: A systematic review. Am J Orthod Dentofacial Orthop. 2019;155(3): 313-29. DOI: 10.1016/j.ajodo.2018.10.015.

18. Topkara A. External apical root resorption caused by orthodontic treatment: a review of the literature. Eur J Paediatr Dent. 2011;12(3):163-6.

19. Lunardi D, Bécavin T, Gambiez A, Deveaux É. Orthodontically induced inflammatory root resorption: apical and cervical complications. J Dentofacial Anom Orthod 2013;16:102. DOI: 10.1051/odfen/2012402. 
20. Pastro JDV, Nogueira ACA, Salvatore de Freitas KM, Valarelli FP, Cançcado RH, et al. Factors associated to apical root resorption after orthodontic treatment. Open Dent J. 2018;12:331-39. DOI: 10.2174/1874210601812010331.

21. Lund H, Gröndahl K, Hansen K, Gröndahl HG. Apical root resorption during orthodontic treatment. A prospective study using Cone Beam CT. Angle Orthod. 2012;82(3):480-7. DOI: 10.2319/061311-390.1.

22. Marques LS, Martins-Júnior PA, Ramos-Jorge ML, Paiva SM. Root resorption in orthodontics: An evidence-based approach. En: Bourzgui F. Orthodontic Basic Aspects and Clinical Considerations. Croatia: Intech Open; 2012. p. 429-446.

23. Mehta SA, Deshmukh SV, Sable RB, Patil AS. Comparison of 4 and 6 weeks of rest period for repair of root resorption. Prog Orthod. 2017;18(1):18. DOI: 10.1186/ s40510-017-0173-1.

24. González F, Robles V, Rivero L, Palis M, Pulido J. Reabsorción radicular inflamatoria en sujetos con tratamiento ortodóncico. Cartagena (Colombia). Salud Uninorte. 2012;28(3):382-90.

25. Krishnan V. Root resorption with orthodontic mechanics: pertinent areas revisited. Aust Dent J. 2017;62 Suppl 1:71-7. DOI: 10.1111/adj.12483.

26. Fernandes LQP, Figueiredo NC, Antonucci CCM, Lages EMB, Andrade Jr I, Capelli Junior J. Predisposing factors for external apical root resorption associated with orthodontic treatment. Korean J Orthod 2019;49(5):310-8. DOI: 10.4041/kjod.2019.49.5.310.

27. Fang X, Qi R, Liu C. Root resorption in orthodontic treatment with clear aligners: A systematic review and meta-analysis. Orthod Craniofac Res. 2019;22(4):259-69. DOI: $10.1111 /$ ocr.12337.

28. Weiland F. Fuerzas de ortodoncia y reabsorciones radiculares: una revisión. Rev Esp Ortod. 2010;40:69-74.

29. Roscoe MG, Meira JB, Cattaneo PM. Association of orthodontic force system and root resorption: A systematic review. Am J Orthod Dentofacial Orthop. 2015;147(5):610-26. DOI: 10.1016/j.ajodo.2014.12.026.

30. Quintero B, Yepes E, Rendón J. Reacciones del tejido pulpar a movimientos ortodóncicos específicos: una revisión de la literatura. Revista Nacional de Odontología. 2011;7(13):54-60.

31. Motokawa M, Sasamoto T, Kaku M, Kawata T, Matsud Y, et al. Association between root resorption incident to orthodontic treatment and treatment factors. Eur J Orthod. 2012;34(3):350-6. DOI: 10.1093/ejo/cjr018.

32. Iglesias-Linares A, Sonnenberg B, Solano B, Yañez-Vico RM, Solano E, Lindauer SJ, et al. Orthodontically induced external apical root resorption in patients treated with fixed appliances vs removable aligners. Angle Orthod. 2017;87(1):3-10. DOI: 10.2319/02016-101.1.

\section{Correo electrónico de los autores:}

Daniela Alejandra Torres-Pinto: tanitorresp91@gmail.com María Catalina Álvarez-Parker: dra.calvarezp@gmail.com 